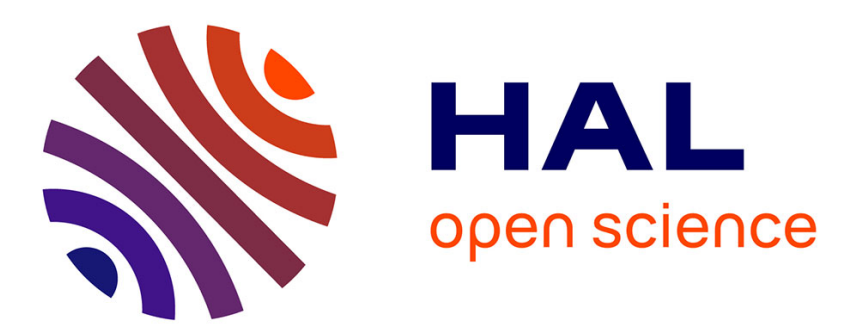

\title{
Human squat motion: joint torques estimation with a 3D model and a sagittal model
}

O Bordron, C Huneau, E Le Carpentier, Yannick Aoustin

\section{To cite this version:}

O Bordron, C Huneau, E Le Carpentier, Yannick Aoustin. Human squat motion: joint torques estimation with a 3D model and a sagittal model. New trends in medical and service robotics (Springer), pp.247-255, 2020. hal-03000335

\section{HAL Id: hal-03000335 \\ https://hal.science/hal-03000335}

Submitted on 11 Nov 2020

HAL is a multi-disciplinary open access archive for the deposit and dissemination of scientific research documents, whether they are published or not. The documents may come from teaching and research institutions in France or abroad, or from public or private research centers.
L'archive ouverte pluridisciplinaire HAL, est destinée au dépôt et à la diffusion de documents scientifiques de niveau recherche, publiés ou non, émanant des établissements d'enseignement et de recherche français ou étrangers, des laboratoires publics ou privés. 


\title{
Human squat motion: joint torques estimation with a 3D model and a sagittal model.
}

\author{
O. Bordron, C. Huneau, E. Le Carpentier, and Y. Aoustin \\ Laboratoire des Sciences du Numérique de Nantes, UMR CNRS 6004, \\ Centrale Nantes, Université de Nantes, \\ 1 rue de la Noë, 44321 Nantes Cedex 3, France, \\ surname.nameduniv-nantes.fr
}

\begin{abstract}
A specific half squat motion is analyzed as a planar movement. Experimental data about this motion are recorded with a motion capture device and two force plates. Joint torques are estimated with -a 3D Opensim model and a sagittal Matlab model. Torque variations from the two models are consistent even if the magnitudes of the corresponding variables differ. These results are attributable to the different anthropometric tables which the two models are based on. Another strategy developed here consists in estimating joint torques without the measured ground reaction forces. In that case, global vertical reaction force is well estimated. The use of the sagittal Matlab model is an efficient way to preliminarily analyze squat trajectories. The next step in this work is the study of the influence of the load represented by the weight of a knee prosthesis.
\end{abstract}

Keywords: Squat motion - Dynamic model $\cdot$ Motion capture $\cdot$ Force plate.

\section{Introduction}

A repetitive movement over a long period of time, habituation to poor posture, handling heavy load can lead to musculoskeletal disorders (MSDs). They are the leading cost drivers in the workers compensation system [5]. The most important thing is to prevent MSDs by automating repetitive tasks. Another solution is to assist the operator with a passive exoskeleton [2] to maintain posture or active [1], [8]. But it is also necessary to help a patient with rehabilitation when a MDS has appeared [13]. Regarding the human musculoskeletal system of movement analyzes, joint torques developed by muscles are useful to prevent or treat MSDs. Often for these analyzes the squat motion is routinely prescribed by physical therapists and sport medicine physicians [3]. Authors demonstrate a technique to calculate the EMG instantaneous median frequency to assess muscle fatigue during a dynamic exercise commonly prescribed in patients with an anterior cruciate ligament deficiency. A lot of studies are based on the squat movement. Without being exhaustive we can mention the following interesting works. Hwang et al [7] show that for two different symmetrical lifting techniques squat and stoop, there are no significant differences in maximum lumbar joint moments. Bonnet et al [4] estimate in real-time the lower-limb joint and torso kinematics by using a single inertial measurement unit placed on the lower back. Wei et al [11] compare the kinematics of young and elderly subjects during normal squatting activity. They observe that the elderly enjoys 
more hip flexion/extension angles than the young, with the squatting posture assumed. Their purpose is to establish a standard of designing lower extremity prosthesis.

These studies focus mainly on the kinematics of movement. However, there is no investigation into the estimation of joint torques developed by muscles. The knowledge of these torques is important to evaluate the possibilities of assisting a joint with an orthosis for example. Our study is also based on a squat movement. Considering that this movement takes place in the sagittal plane our strategy is to evaluate the torques with a simplified sagittal model as follows. During the squat experimental data are recorded with a motion capture device and two force plates. An Opensim Model allows us to estimate the position, velocity and acceleration of the ankle, knee an hip joints of the human subject. The main advantage of musculoskeletal models is to provide a noninvasive means to study human movement [10], [6]. Then a simplified inverse dynamic model of a biped, taking into account explicitly the contact with the ground, is designed. Several cases are considered to compare the articular torques calculated thanks to a sagittal model with the torques estimated with OpenSim. Our objective is to define an efficient but simple tool, which is based on the dynamical effects of the lower-limbs to size an orthosis. This paper is outlined as follows. Section 2 presents the used material and the methods. Section 3, the results are analyzed. Section 4 a discussion is conducted about the assumption symmetric effort distribution for the sagitttal model. Section 5 offers our conclusion and perspectives.

\section{Materials and Methods}

\subsection{Squat motion}

The squat movement studied is defined as a four-step cycle so that it can be repeated in a continuous loop by the subject. The total duration of that cycle is $T=4 s$ and each step lasts one second. In its initial state, the subject stands upright with his knees slightly bent (Fig. 1a)). The arms are stretched horizontally. The trunk is also slightly inclined with respect to the longitudinal axis. The cycle is defined as follows:

1. The subject goes down bending his knees while keeping his arms straight and his trunk slightly inclined forward. At the end of this step, the subject's thighs are parallel to the ground in order to perform a half squat (Fig. 1b)).

2. This pose is then maintained before starting the ascent step.

3. The subject returns to the initial pose (Fig. 1c)).

4. The initial pose is maintained.

\subsection{Data acquisition}

The Motion Capture experiment was conducted with a 25-year-old woman, height $\mathrm{H}=$ $1.73 \mathrm{~m}$ and mass $\mathrm{M}=62 \mathrm{~kg}$. A set of 17 marker arrays were placed on the subject's head, trunk, arms, legs and feet. During the movement, the spatial coordinates of the marker arrays were estimated by the ART IR acquisition system using eight cameras with an acquisition frequency of $60 \mathrm{~Hz}$. Two force plates were used to measure the center of 


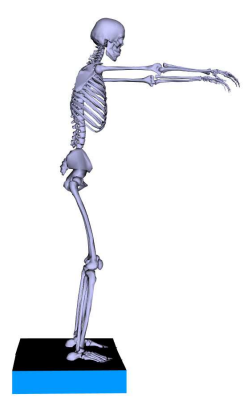

a)

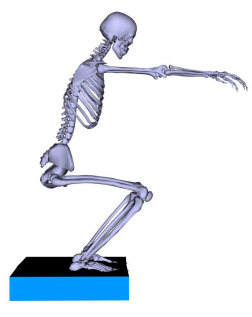

b)

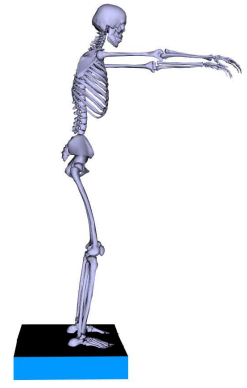

c)

Fig. 1: a) Initial configuration of biped. b) Intermediate configuration. c) Final configuration - initial one in the next squat motion.

pressure of each foot of the subject on the ground as well as the ground reaction forces and moments. $N=20$ squats were performed by the subject according to the motion defined in 2.1. To eliminate the measurement noise, we applied a Butterworth filter to all signals with a cut-off frequency of $5 \mathrm{~Hz}$. From the data of the force plates and more specifically the global vertical ground reaction, a rupture detection method -the CUSUM algorithm [9]- was employed to detect initial cycle times. All data presented in Section 3 were averaged with respect to the $N$ cycles.

\subsection{Opensim Model}

The model developed on the software Opensim is based on the Rajagopal model [10]. It is a complete musculoskeletal model designed to perform dynamic simulations of human movement. For the dynamic analysis of squat movement, this model has been adapted with unilateral constraints between the ground and both feet so that they are in full flat contact with the ground (Fig. 2). Virtual markers are defined in respect to the different bodies of the model.

The estimation of the articular torques is done in three steps. First, a scaling step is required to adjust the size, mass distribution and position of the Opensim model's virtual markers. From a static trial, the position data of the experimental markers are recorded via the motion capture system. Then, the deviation between the experimental markers and their corresponding virtual marker is minimized in the sense of least squares. The optimization variables are the segment lengths.

Then, several squat trials are carried out by the subject. The position of the experimental markers are imported on Opensim. The inverse kinematic step consists in minimizing a least squares problem in order to model the performed motions. Finally, the inverse dynamic step enables the joint torques estimation from the forces measured by the force plates and the motions obtained by the inverse kinematic model. 


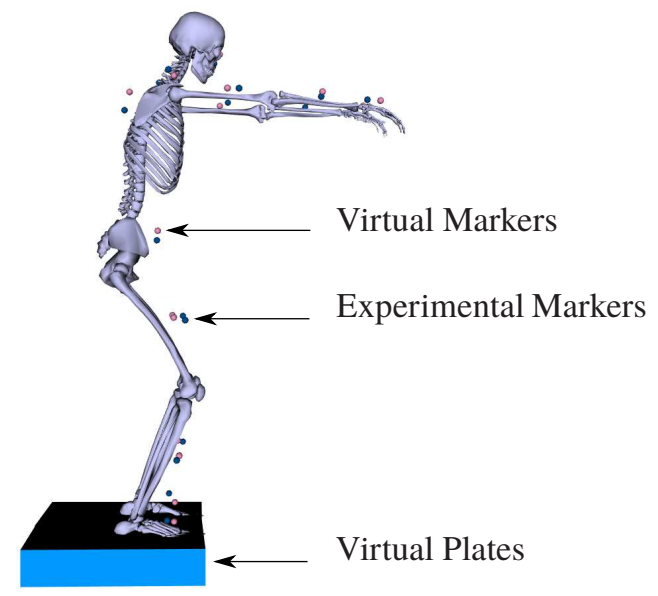

Fig. 2: Full-Body Musculoskeletal Opensim Model for the squat movement.

\subsection{Matlab Model}

Anthropomorphic parameters A sagittal model of a nine-link planar biped is designed in order to evaluate the joint torques, which are developed during the squat motion. The physical parameters of this model are obtained from Winter's anthropometric table [12] by considering the height and weight of the subject. The planar biped has a trunk, two arms, two thighs, two shins and two feet, see Fig. 3. For this planar biped table 1 gathers the length, mass, center of mass position and inertia moment of each segment.
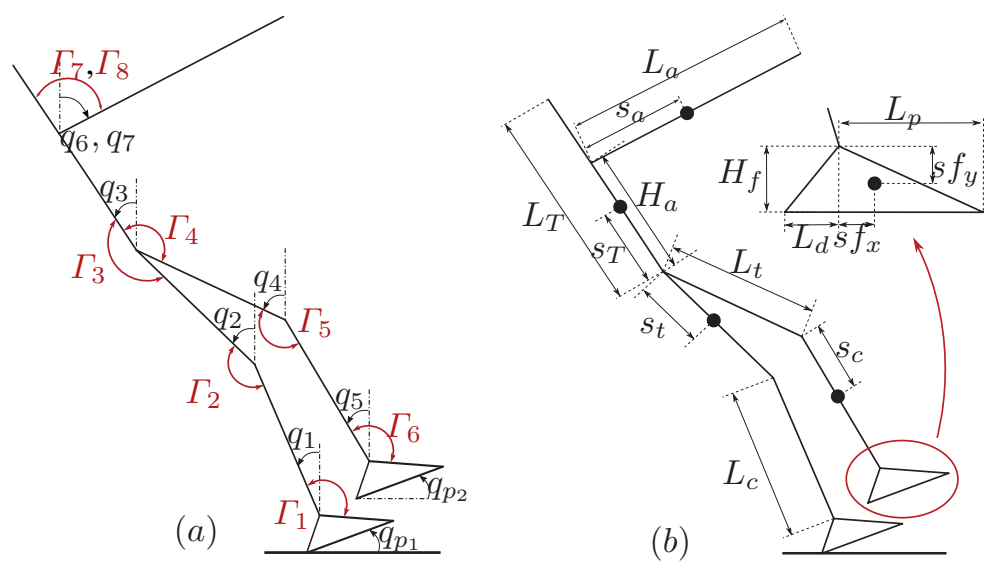

Fig. 3: Model of the planed biped. a) Parameterization of the biped. Note that angles are positive in counterclockwise. b) Length segments and position of centers of mass. 


\begin{tabular}{|l|c|c|c|c|c|}
\hline & Foot & Calf & Thigh & Trunk & Arm \\
\hline Segment Weight $(\mathrm{kg})$ & $\mathrm{m}_{\mathrm{f}}=0.90$ & $\mathrm{~m}_{\mathrm{c}}=2.88$ & $\mathrm{~m}_{\mathrm{t}}=6.20$ & $\mathrm{~m}_{\mathrm{T}}=35.8$ & $\mathrm{~m}_{\mathrm{a}}=3.10$ \\
Segment Length $(\mathrm{m})$ & $\mathrm{L}_{\mathrm{f}}=0.068$ & $\mathrm{~L}_{\mathrm{c}}=0.426$ & $\mathrm{~L}_{\mathrm{t}}=0.424$ & $\mathrm{~L}_{\mathrm{T}}=0.813$ & $\mathrm{~L}_{\mathrm{a}}=0.761$ \\
& $\begin{array}{l}\mathrm{L}_{\mathrm{d}}=0.196 \\
\mathrm{H}_{\mathrm{f}}=0.068\end{array}$ & & & $\mathrm{H}_{\mathrm{a}}=0.498$ & \\
Inertia $\left(\mathrm{kg} \cdot \mathrm{m}^{2}\right)$ & $\begin{array}{l}\mathrm{I}_{\mathrm{f}}=0.0087 \\
\text { Center of mass (m) }\end{array}$ & $\mathrm{I}_{\mathrm{c}}=0.0476$ & $\mathrm{I}_{\mathrm{t}}=0.1162$ & $\mathrm{I}_{\mathrm{T}}=2.2508$ & $\mathrm{I}_{\mathrm{a}}=0.1385$ \\
& $\mathrm{sf}_{x}=0.098$ & $\mathrm{~s}_{\mathrm{c}}=0.184$ & $\mathrm{~s}_{\mathrm{t}}=0.184$ & $\mathrm{~s}_{\mathrm{T}}=0.329$ & $\mathrm{~s}_{\mathrm{a}}=0.304$ \\
\hline
\end{tabular}

Table 1: Anthropomorphic parameters of the human planar model.

The biped configuration is described with q, Fig. 3:

$$
\mathbf{q}=\left[q_{1}, q_{2}, q_{3}, q_{4}, q_{5}, q_{6}, q_{7}, q_{\mathrm{p}_{1}}, q_{\mathrm{p}_{2}}, x_{\mathrm{h}}, y_{\mathrm{h}} \cdot\right]^{\top}
$$

Unilateral constraints between the ground and the stance foot For the studied squat motion, both feet have a flat contact with the ground. In the sagittal plane, the action of the ground on each foot can be modeled as a wrench with three components, expressed at $\mathrm{A}_{i}$, as illustrated in Fig. 4 such as:

$$
\mathbf{r}_{i}=\left[\begin{array}{lll}
r_{i x} & r_{i y} & M_{i z}
\end{array}\right]^{\top}, \text { for } i \in\{1,2\}
$$

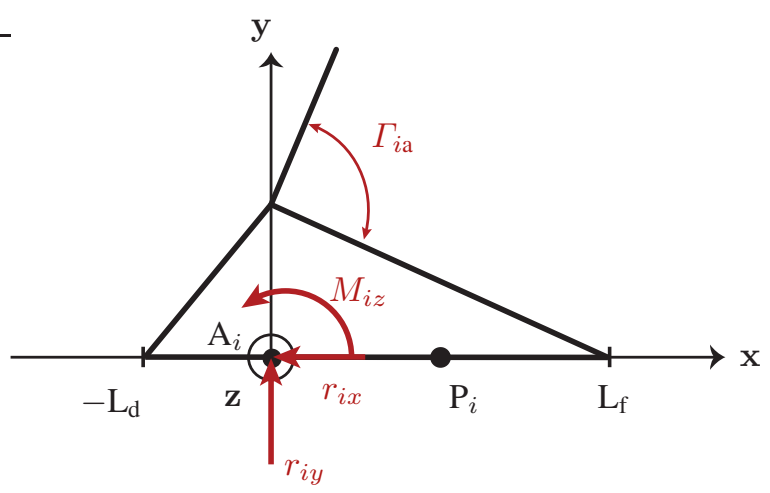

Fig. 4: Torques and forces acting on the foot.

The conditions of feet flat contact on the ground are as follows:

$$
\left[x_{\mathrm{A}_{i}} y_{\mathrm{A}_{i}} q_{\mathrm{p}_{i}}\right]^{\top}=\left[0-\mathrm{H}_{\mathrm{f}} 0\right]^{\top} \text {, for } i \in\{1,2\}
$$

By deriving (2) in time, we obtain the conditions in velocity and acceleration of each foot and the ground, expressed in point $\mathrm{A}_{i}$ :

$$
\mathbf{J}_{i} \dot{\mathbf{q}}=\mathbf{0}_{3 \times 1} \text { and } \mathbf{J}_{i} \ddot{\mathbf{q}}+\dot{\mathbf{J}}_{i} \dot{\mathbf{q}}^{2}=\mathbf{0}_{3 \times 1} \text {, for } i \in\{1,2\} \text {. }
$$


The non-rotation of the supporting feet reflects the dynamic equilibrium of the feet during the squat motion. This state can be described by the trajectory of the centre of pressure $(\mathrm{CoP})$ of each foot. This point, called $\mathrm{P}_{i}$, represents the application point of the ground reaction forces such as $M_{i z}=0$, for $i \in\{1,2\}$. That definition leads to:

$$
x_{\mathrm{P}_{i}}=\frac{\Gamma_{i \mathrm{a}}+\mathrm{m}_{\mathrm{f}} \mathrm{gsf}_{x}-\mathrm{H}_{\mathrm{f}} r_{i x}}{r_{i y}} \text { and }-\mathrm{L}_{\mathrm{d}} \leq x_{\mathrm{P}_{i}} \leq \mathrm{L}_{\mathrm{f}} \text {, for } i \in\{1,2\}
$$

The condition of dynamic equilibrium of the feet can be expressed by (4).

Dynamic model The dynamic model of the nine-links planar model is defined as follows:

$$
\mathbf{D}(\mathbf{q}) \ddot{\mathbf{q}}+\mathbf{C}(\mathbf{q}, \dot{\mathbf{q}}) \dot{\mathbf{q}}+\mathbf{G}(\mathbf{q})=\mathbf{B} \boldsymbol{\Gamma}+\mathbf{J}_{1}^{\top} \mathbf{r}_{1}+\mathbf{J}_{2}^{\top} \mathbf{r}_{2}
$$

Here $\Gamma_{8 \times 1}$ is the torque vector, the $3 \times 1$ ground reaction wrenches $\mathbf{r}_{1}$ and $\mathbf{r}_{2}$ are applied to respectively the feet 1 and $2 . \mathbf{B}_{11 \times 8}$ is a constant matrix to take into account of the influence the torques in each joint. $\mathbf{J}_{1}^{\top}$ and $\mathbf{J}_{2}^{\top}$ are the $11 \times 3$ transposed Jacobian matrices, which convert ground reaction wrenches at feet 1 and 2 in torques applied to the $\mathrm{d}$ joints. $\mathbf{D}_{11 \times 11}$ is the symmetric positive inertia matrix, $\mathbf{C}_{11 \times 11}$ represents the Coriolis and centrifugal effects, and $\mathbf{G}_{11}$ defines the gravity effect.

Calculation of $\Gamma, r_{1}$, and $r_{2}$ By the motion capture acquisition system, kinematic data related to the squat motion were recorded for each sampling time. Consequently, the left part of (5) can be calculated. The unknown variables are the 8 joint torques and the $2 \times 3$ components of $\mathbf{r}_{1}$ and $\mathbf{r}_{2}$. Then, the global model can be considered as a system with 11 equations and 14 unknown variables. By considering the sagittal plane as a symmetric plane for the motion and the distribution of masses, let us consider that $\mathbf{r}_{1}=\mathbf{r}_{2}=\mathbf{r}$. In this way, the inverse dynamic model can be totally resolved by using (6). The matrix $\left[\mathbf{B}\left(\mathbf{J}_{1}^{\top}+\mathbf{J}_{2}^{\top}\right)\right]$ has always been invertible in our numerical tests.

$$
\left[\begin{array}{c}
\mathbf{\Gamma} \\
\mathbf{r}
\end{array}\right]=\left[\mathbf{B}\left(\mathbf{J}_{1}^{\top}+\mathbf{J}_{2}^{\top}\right)\right]^{-1}(\mathbf{D}(\mathbf{q}) \ddot{\mathbf{q}}+\mathbf{C}(\mathbf{q}, \dot{\mathbf{q}}) \dot{\mathbf{q}}+\mathbf{G}(\mathbf{q}))
$$

Estimation $\hat{\Gamma}$ of the joint torques by the least mean squares estimation The ground reaction forces applied to feet 1 and 2 are recorded with two force plates. These data can be used to inverse (5). The global system has more equations than unknown variables. The least mean squares estimation allows us to estimate the joint torques $\hat{\Gamma}$ with (7), where $\mathbf{B}^{+}$is the $8 \times 11$ pseudo-inverse matrix of $\mathbf{B}$.

$$
\hat{\boldsymbol{\Gamma}}=\mathbf{B}^{+}\left(\mathbf{D}(\mathbf{q}) \ddot{\mathbf{q}}+\mathbf{C}(\mathbf{q}, \dot{\mathbf{q}}) \dot{\mathbf{q}}+\mathbf{G}(\mathbf{q})-\mathbf{J}_{1}^{\top} \mathbf{r}_{1}-\mathbf{J}_{2}^{\top} \mathbf{r}_{2}\right)
$$

\section{Results}

\subsection{Kinematic and force plates data of the squat motion}

The data resulting from the motion capture session were imported into Opensim. The inverse kinematic model was solved according to Section 2.3 to obtain angular variable 
trajectories for each joint. For the use of the sagittal model, motion trajectories have been projected in the sagittal plane. The analysis of the measured average trajectory of the $\mathrm{CoP}$ shows the presence of a privileged direction. The trajectory of the global CoP -the average of the trajectories of the CoPs for the left and right foot- evolves mainly along an axis parallel to the $\mathrm{x}$ axis. This result is consistent with the fact that the squat movement is a plane-type movement.

\subsection{Comparison of the estimation of the joint torques between a 3D Opensim model and a sagittal Matlab model.}

From the motion obtained in Section 3.1, inverse dynamic model has been first resolved with the 3D Opensim model. From the projected trajectories in the sagittal plane, the resolution was carried out by using (7). Figure 5 illustrates the results obtained from both resolutions. The torque estimation made by the two models at the ankle and knee joints are almost the same for both left and right sides. Nevertheless, the hip torque estimation made by the Matlab model differs from the Opensim model. Variations in the anthropomorphic data used in both models can lead to these differences. The few torques variations of both shoulders are due to the gravity effect in order to maintain a stable position.

\section{Discussion}

In a simulation approach, to study other squat trajectories, it may be interesting to solve the dynamic model by freeing us from the measured data. In this case, an assumption on the efforts distribution is necessary. As shown with (6), joint torques and reaction forces can be calculated by assuming that the subject distributes the forces equally over each of his feet. The magnitude of the vertical reaction is observed greater for the right foot than for the left one in average. This means that the subject uses the right foot to a higher extent during the experiment. Consequently, the assumption of equally distribution on both feet is not valid. Nevertheless, the calculation shows that the global vertical reaction is quite well estimated in average. The use of the sagittal Matlab model combined with the extracted kinematic data is in accordance with the global external forces measured. Under the assumption symmetric effort distribution, the calculated joint torques are the same at the left side of the human model than at the right one.

\section{Conclusion}

The results show a coherence between a 3D model and a 2D model for a squat motion. It highlights a variability according to the anthropometric data used. From the 2D model, it is possible to solve the inverse dynamic model by making an assumption on the distribution of forces. These results underline the interest of using a planar model for a squat movement. The perspective is to investigate the influence of the load represented by the weight of a knee prosthesis. 
O. Bordron et al.

Opensim model [N.m]
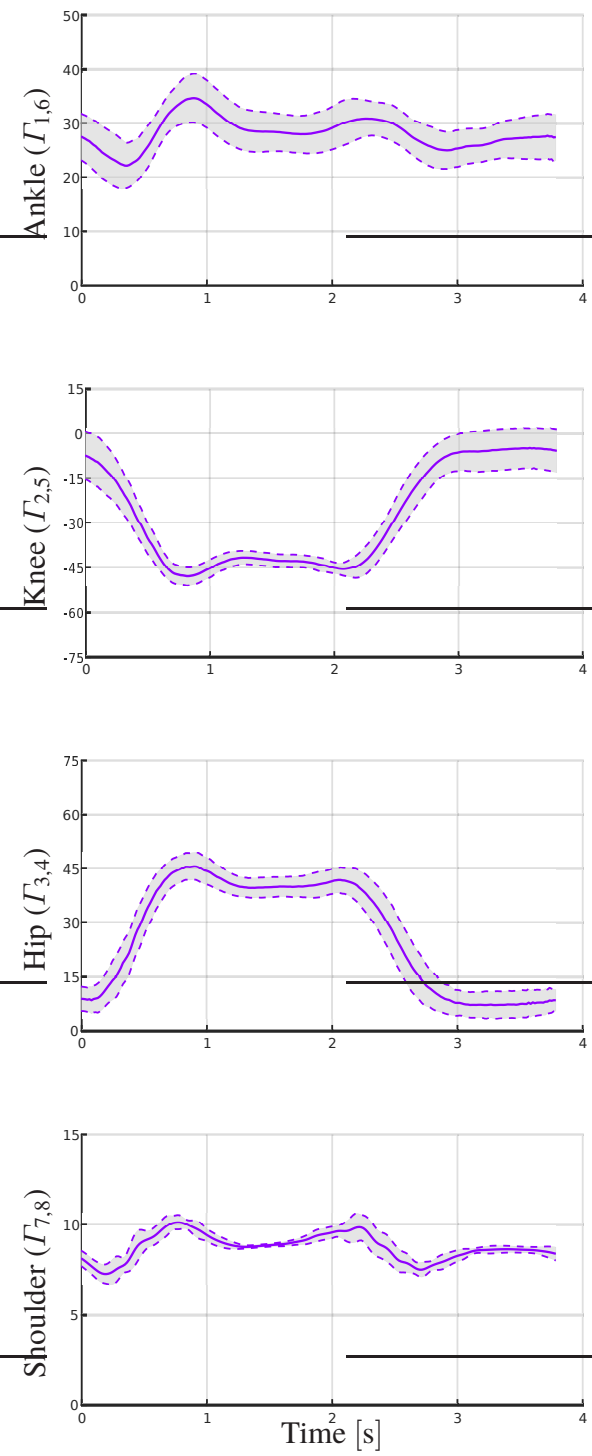

Matlab model [N.m]
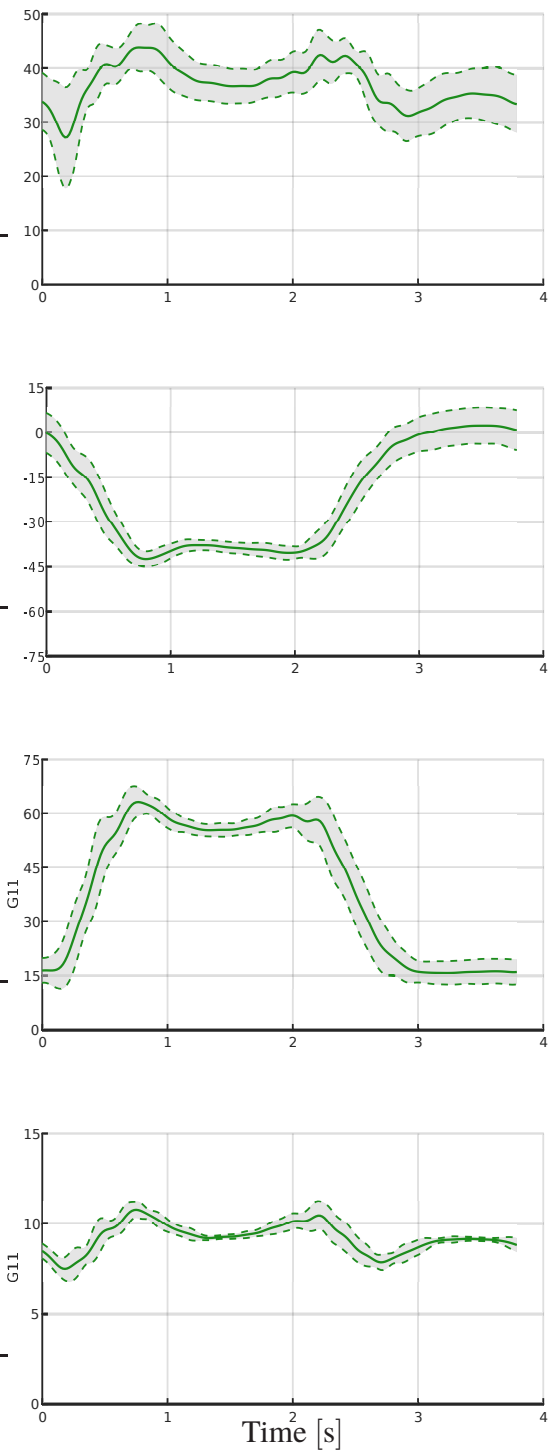

Fig. 5: Joint torques calculated by the Matlab model (green lines) and estimated by the Opensim model where left and right sides have been averaged (violet lines).

\section{References}

1. Y. Aoustin. Walking gait of a biped with a wearable walking assist device. Int. J. of Humanoid Robotics, 12(2):1550018, 2015. 
2. Y. Aoustin and A. Formalskii. Walking of biped with passive exoskeleton: evaluation of energy consumption. Multibody System Dynamics, 43(1):71-96, 2018.

3. P. Bonato, M. S. Cheng, J. Gonzalez-Cueto, A. Leardini J. O'Connor, and S. H. Roy. Emgbased measures of fatigue during a repetitive squat exercice. IEEE Engineering in medicine and biology Magazine, 20(6):133-143, 2018.

4. V. Bonnet, C. Mazzà, and P. Fraisse. Real-time estimate of body kinematics during a planar squat task using a single inertial measurement unit. IEEE Trans. on Biomedical engineering, 60(7):1920-1926, 2013.

5. M. R. Cusimano-Reaston and B. Carney. Legal changes necessitate proactive management of musculokeletal disorders: The role of electrodiagnostic functional assessment soft tissue management program. In Proc. of 33rd Annual international conference of IEEE EMBS, pages 7570-7573, Boston, Massachusetts USA, 2011.

6. G. Durandau, D. Farina, and M. Sartori. Robust real-time musculoskeletal modeling driven by electromyograms. IEEE Trans. on Biomedical engineering, 65(3):556-564, 2018.

7. S. Hwang, Y. Kim, and Y. Kim. Joint kinetics and lumber curvatures during symmetric lifting: squat and stoop. In Proc. of Int. Conf. on BioMedical Enginneering and Informatics, volume 8, pages 818-822, Sanya, Hainan China, 2008.

8. H. Kazerooni and R. Steger. The berkeley lower extremity exoskeleton. Trans. ASME J. Dynamic Systems Measurement and Control, 128(1):14-25, 2006.

9. E. S. Page. Continuous inspection schemes. Biometrika, 41(1-2):100-115, 061954.

10. A. Rajagopal, C. L. Dembia, M. S. DeMers, D. D. Delp, J. L. Hicks, and S. L. Delp. Fullbody musculoskeletal model for muscle-driven simulation of human gait. IEEE Trans. on Biomedical engineering, 63(10):2068-2079, 2016.

11. S. Wei, H. Zhou, X. Li, and C. Wang. Kinematics of lower extremity for the young and elderly chinese population during squatting. In Proc. of Int. Conf. on BioMedical Enginneering and Informatics, pages 578-582, Dalian China, 2014.

12. D. A. Winter. Biomechanics and motor control of human movement. Jonhn Wiley and Sons, 2009.

13. A. S. Yusof, A. I.Che-Ani, Z. Hussain, N. Hamzah, R. Boudville, and M.F.A. Rahman. Backdrivability of powered knee orthosis for knee frr swing and knee extension. In Proc. of 7th IEEE Int. Conf. on Control Systems, Computing and Engineering (ICCSCE 2017), pages 331-335, Penang, Malaysia, 2017. 